\title{
Battery-Aware Power Management Based on Markovian Decision Processes
}

\author{
Peng Rong \\ prong@usc.edu \\ Dept. of EE-Systems, University of Southern California \\ Massoud Pedram \\ pedram@ceng.usc.edu
}

Reference [5] also improved on the modeling technique of [3] by using time-indexed semi-Markovian decision processes.

\begin{abstract}
Although the abovementioned DPM techniques can greatly reduce the system power consumption, they are not able to obtain the optimal policy for a battery-powered system. This is because the characteristics of battery behavior were not properly modeled or exploited in these techniques. As demonstrated by research results in [6], the total energy capacity that a battery can deliver during its lifetime is strongly related to the discharge current rate. More precisely, as the discharge current increases, the deliverable capacity of the battery decreases. This phenomenon is called the (current) rate-capacity characteristic. Another important property of batteries, which was analyzed and modeled in [8], is named the relaxation phenomenon (or recovery effect). It is caused by the concentration gradient of active materials in the electrode and electrolyte formed in the discharge process. Driven by the concentration gradient, the active material at the electrolyte-electrode interface, which is consumed by the electrochemical reactions during discharge, is replenished with new active materials through diffusion. Thus the battery capacity is somewhat recovered during a no-use state. Due to these non-linear characteristics, a minimum power consumption policy does not always necessarily result in the longest battery service life because the energy capacity of its power sources may be not fully exploited when the cut-off voltage of the battery is reached.
\end{abstract}

A series of battery models have been proposed. These can be divided into two categories: electrochemical model and stochastic model. The electrochemical models are based on diffusion equations and provide an accurate description of the underlying electrochemical process. A low level model for lithium-ion batteries and a high level model for the time-varying load were proposed in [8] and [9], respectively. The electrochemical models require a predetermined workload profile. However, in most real situations, the workload is unknown a priori and often evolves as a random process. In these cases, stochastic models are needed. Stochastic models describe the battery behavior as a stochastic process whose parameters are extracted from the electrochemical characteristics of the simulated battery. Some stochastic models have been reported in the literature, e.g. a discrete-time VHDL model [10] and a discretetime Markovian chain model [11]. The stochastic model in [11] is a Markovian chain of the battery states of charge with forward and backward transitions corresponding to the normal discharge and recovery effect, respectively. The load is expressed as a stochastic demand on the charge units. The model in [11] is mainly focused on the recovery effect. 
A series of battery management policies have been proposed to maximize the battery lifetime. A round robin policy was presented in [11]. Other policies were studied and compared in [12]. A shortcoming of these heuristic approaches is that the optimality cannot be guaranteed. Reference [13] presents a dual-battery power supply structure which consists of two batteries that have different rate-capacity characteristics and uses them in an interleaved manner in responding to different current requirement.

To the best of our knowledge, there has been no reported work for integrating the model of a power-managed portable electronic system with the model of its power source - i.e., batteries. This is, however, precisely the contribution of our paper. More precisely, we extend the work in [4] to achieve a complete model for the battery-powered portable system by introducing and incorporating a new CTMDP model of the battery source. This model correctly captures the two important battery characteristics, i.e., the recovery effect and current-capacity curve. Furthermore, it considers the case of a multiple battery power source with a power switch that is controlled by the power management policy. Based on this model the battery-aware power management problem is formulated as a policy optimization problem based on the CTMDP theory and solved optimally by using linear programming (LP).

This paper targets a power-management portable system as shown in Figure 1. The example depicts a typical dual-battery powered portable system. The system contains a service requestor (SR) to generate the tasks to be serviced, a service provider (SP) which provides the required services, and a service queue (SQ) to store the tasks waiting for service. ${ }^{1}$ The SP is powered by two batteries (B1 and B2), which may have different current-capacity and recovery characteristics. B1 and B2 alternately discharge and provide power for the SP. The power switch (SW) selects either B1 or B2 to provide power at any given time. Note that only one of the batteries is used at a given time and the other is always resting at that time. Based on this model, we will show that an optimal management scheme can be obtained by solving a LP problem.

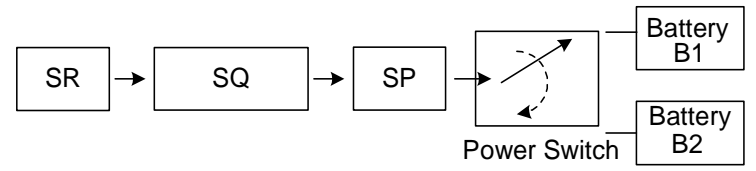

Figure 1. Dual-battery powered portable system model.

The paper is organized as follows: The model of the batterypowered portable power-managed system is described in Section 2. The solution technique for the optimal problem is described in Section 3. In Section 4, we present the experiment results and we conclude in Section 5.

\section{SYSTEM MODELING}

First, we model each component in this portable system. Next, based on these models we build the complete model of the powermanaged, portable, battery-powered system.

The models of the SR, SQ and SP are similar to those described in [4]. These components are all modeled as stationary continuous-

\footnotetext{
${ }^{1}$ Notice that it is straight-forward to handle multiple SR's, multiple SP's and even multiple SQ's. In this paper, we focus on single $\mathrm{SR}$, single SP, and single SQ to simplify the presentation.
}

time Markovian decision processes. Figure 2 gives examples of each of the SR, SQ and SP models.

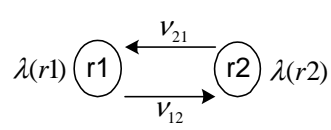

(a) SR

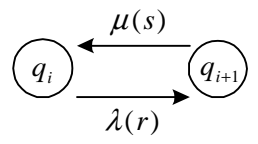

(b) $\mathrm{SQ}$

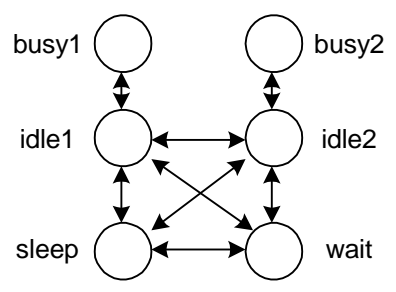

(c) SP

Figure 2. CTMDP models of the SR, SQ and SP.

The SR model consists of a state set $\mathbf{R}=\left\{r_{i}, i=0,1,2, \ldots, R\right\}$ and a generator matrix $G_{S R}$, where $\mathrm{R}$ is the number of the states of the SR. The SQ model consists of a state set $\mathbf{Q}=\left\{q_{i}, i=0,1,2, \ldots, Q\right\}$ and a generator matrix $G_{S Q}(r, s)$, where $\mathrm{Q}$ is the maximum length of the queue, $s$ denotes a state of the SP, and $r$ denotes a state of the SR. The SP model consists of a state set $\mathbf{S}=\left\{s_{i}\right.$ s.t. $\left.i=1,2, \ldots, S\right\}$, an action set $A_{s}$, and a parameterized generator matrix $G_{S P}\left(a_{s}\right)$, where $a_{s} \in A_{s}$. The SP can be described by a quadruple $\left(\chi, \mu(s), \operatorname{pow}(s)\right.$, ene $\left.\left(s_{i}, s_{j}\right)\right)$, where $\chi$ is the transition speed matrix of the SP. $\mu(s)$ is the service speed of the SP when it is in state $\mathrm{s}, \operatorname{pow}(s)$ is the power consumption of the SP staying in state $s$ and ene $\left(s_{i}, s_{j}\right)$ is the energy required by the SP to transit from state $s_{i}$ to $s_{j}$. There are two kinds of transition: autonomous and command-controlled transition. A command-controlled transition may only occur when the SP receives a command from the DPM controller that asks the SP to make such a transition, e.g. the transition from state idlel to state busyl. An autonomous transition takes place without instruction from the DPM controller, e.g. the transition from state busyl to state idle1, which takes place autonomously when the SP finished the current service.

The expected power consumption (cost rate) of the SP when it is in state $s$ and action $a_{s}$ is chosen is calculated as

$$
\operatorname{pow}^{\prime}\left(s, a_{s}\right)=\operatorname{pow}(s)+\frac{1}{\tau_{s}^{a_{s}}} \sum_{\substack{s^{\prime} \neq s \\ s \in S}} p_{s, s^{\prime}} a_{s} \operatorname{ene}\left(s, s^{\prime \prime},(2-1)\right.
$$

where $\tau_{s}^{a_{s}}=1 / \sum_{s^{\prime} \in S} \sigma_{s, s^{\prime}}^{a_{s}}$, represents the average time that the SP stays in the state $\mathrm{s}$, whereas

$$
P_{s, s^{\prime}}^{a_{s}}=\frac{\sigma_{s, s^{\prime}}^{a_{s}}}{\sum_{s^{\prime}} \sigma_{s, s^{\prime}}^{a_{s}}}
$$

represents the probability that the next state of the SP is $s^{\prime}$ when its present state is $s$ and action $a_{s}$ is chosen. 


\subsection{Model of the Power Switch}

The Power Switch (PS) is modeled as a stationary, continuous-time Markovian process, with a state set $\mathbf{W}=\left\{w_{i}\right.$ s.t. $\left.i=1,2, \ldots, W\right\}$, an action set $A_{s w}=\left\{a_{s w}(i)\right.$ s.t. $\left.i=1,2, \ldots, W\right\}$, and a generator matrix $G_{S W}$. Here $a_{s w}(i)$ means that the $i_{t h}$ battery source should be used next to power the system.

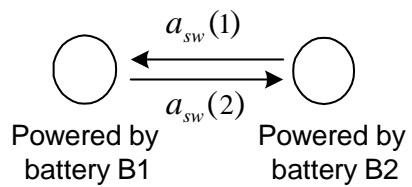

Figure 3. CTMDP model of the PS.

\subsection{Model of the Battery}

The battery (BAT) is modeled as a stationary, continuous-time Markovian decision process with a state set $\mathbf{B}=\left\{b_{i}, r s_{i}\right.$ s.t. $\left.i=0,1,2, \ldots, N\right\}$, a parameterized generator matrix $G_{B}(s, w, b)$, and a function $e n e\left(b_{i}, b_{j}\right): N \times N \rightarrow R$.

The subscript $i$ of state $b_{i}$ or $r s_{i}$ in the state set $\mathbf{B}$, denotes that in this state the remaining energy capacity of the BAT is $\frac{i}{N} \times 100 \%$ of the full energy capacity. Therefore, $b_{0}$ implies that the battery has been completely discharged whereas $b_{N}$ means that the battery is fully charged. For example, ignoring the states $r s_{i}$ for the moment, let $N=5$, then the state set is $\mathrm{B}=\{0,20 \%, 40 \%, 60 \%, 80 \%, 100 \%\}$. Function ene $\left(b_{i}, b_{j}\right)$ represents the energy-capacity difference between state $b_{i}$ and $b_{j}$. Figure 4 illustrates the CTMDP model of the BAT.

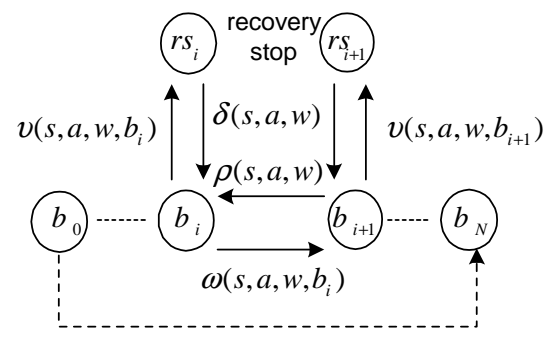

Figure 4. CTMDP model of the BAT.

In this model, state $b_{i}$ represents an "active" state, in which the battery may be discharged when it is used or can recover capacity when it is resting.

State $r s_{i}$ represents a "stable" state, in which the battery can neither discharge nor recover.

The transition from state $b_{i+1}$ to $b_{i}$ represents the discharge process of the battery, $\rho(s, a, w)$ denotes the transition rate. If PS selects this battery and SP consumes power when it is in state $S$ and action $a$ is chosen, the value of $\rho(s, a, w)$ is determined by equation (2-1) (cf. Table 1); otherwise, $\rho(s, a, w)$ is equal to 0 .
The transition from state $b_{i}$ to $b_{i+1}$ represents the recovery process of the battery, $\omega\left(s, a, w, b_{i}\right)$ denotes the transition rate, which is a function of the SP state $S$ and the battery state $b_{i}$. If the SP does not consume power when it is in state $S$ and action $a$ is chosen, or if the PS does not select this battery, the value of $\omega\left(s, a, w, b_{i}\right)$ is determined by the battery state $b_{i}$ (cf. Table 1); otherwise $\omega\left(s, a, w, b_{i}\right)$ is equal to 0.

The transition from state $b_{i}$ to $r s_{i}$ may only occur when the battery is resting. $v\left(s, a, w, b_{i}\right)$ denotes the transition rate. If the SP does not consume power when it stays in state $S$ and action $a$ is chosen, or if the PS does not select this battery, the value of $v\left(s, a, w, b_{i}\right)$ is determined by the battery state $b_{i}$ (cf. Table 1); otherwise $v\left(s, a, w, b_{i}\right)$ is equal to 0.

The transition from state $r s_{i}$ to $b_{i}$ may only occur when the battery is used again. Here $\delta(s, a, w)$ means that if the SP consumes power when it stays in state $S$ and action $a$ is chosen, and if the PS selects this battery, the battery goes from $r s_{i}$ to $b_{i}$ immediately.

The value of $\rho(s, a, w), \quad \omega\left(s, a, w, b_{i}\right), \quad v\left(s, a, w, b_{i}\right)$ and $\delta(s, a, w)$ are summarized in the following table. In the first row of Table 1 , if the SP consumes power when it stays in state $s$ and action $a$ is chosen, $(s, a)$ is set to 1 ; otherwise $(s, a)$ is set to 0 . If the PS selects this battery, $w$ is set to 1 ; otherwise $w$ is set to 0 .

Table 1. Parameters of the BAT

\begin{tabular}{|l|c|c|c|c|}
\hline$(s, a), w$ & 0,0 & 0,1 & 1,1 & 1,0 \\
\hline$\rho(s, a, w)$ & 0 & 0 & $\rho^{\prime}(s, a)^{*}$ & 0 \\
\hline$\omega\left(s, a, w, b_{i}\right)$ & $\omega^{\prime}\left(b_{i}\right)^{* *}$ & $\omega^{\prime}\left(b_{i}\right)^{* *}$ & 0 & $\omega^{\prime}\left(b_{i}\right)^{* *}$ \\
\hline$v\left(s, a, w, b_{i}\right)$ & $v^{\prime}\left(b_{i}\right)^{* *}$ & $v^{\prime}\left(b_{i}\right)^{* *}$ & 0 & $v^{\prime}\left(b_{i}\right)^{* *}$ \\
\hline$\delta(s, a, w)$ & 0 & 0 & $\infty$ & 0 \\
\hline
\end{tabular}

* $\rho^{\prime}(s, a)$ is defined by equation (2-2) in section 2.2.1.

** $\omega^{\prime}\left(b_{i}\right)$ and $v^{\prime}\left(b_{i}\right)$ are functions each defined by a look-up table indexed by $b_{i}$. The actual value of each entry in the two tables is obtained from simulation results. The method is described in more detail in section 2.2.2.

The transition from state $b_{0}$ to $b_{N}$, denoted by the long wraparound dashed arrow line, represents that an exhausted (used-up) battery is replaced with a fresh (fully charged) battery of the same type. This transition is added because without it, state $b_{0}$ becomes a trap. If transition from $b_{0}$ to $b_{N}$ is not included in the model, then when time tends to infinity, the battery will eventually arrive into the state $b_{0}$ and cannot subsequently leave this state. Consequently, no feasible solution would be found when using the LP technique to solve the optimal policy problem.

The battery model is constructed based on the following three assumptions: 
(a) During the discharge process of the battery, only a transition from state $b_{i+1}$ to $b_{i}$ is allowed, where $i=0, \cdots, N-1$, which means that the battery discharges gradually.

(b) When the battery is resting (i.e. not being used), if it is in the state $b_{i}$, it may regain some of its capacity due to the recovery process or it may transit to the state $r s_{i}$. However, when the battery is in the state $r s_{i}$, it cannot recover capacity any more and will stay in this state until it is used again to power up the system. As soon as this happens, the battery moves from state $r s_{i}$ to $b_{i}$ and then possibly to $b_{i-1}$.

(c) During the recovery process of the battery, only a transition from state $b_{i-1}$ to $b_{i}, i=2, \cdots, N$, is allowed, which means that the battery always recovers gradually. State $b_{0}$ means that the battery capacity has been exhausted, so the battery cannot serve any more and should be replaced.

Assumptions (a) and (c) are realistic because of the continuous nature of the electrochemical processes. Assumption (b) also is realistic because the energy recovery speed of a battery diminishes when the rest time increases. Example result depicted in Figure 5 empirically confirms this important observation. Notice that the curve marked with blue ' + ' signs is obtained by simulating a real Liion battery. The horizontal axis denotes the ratio of the rest time to the discharge time.

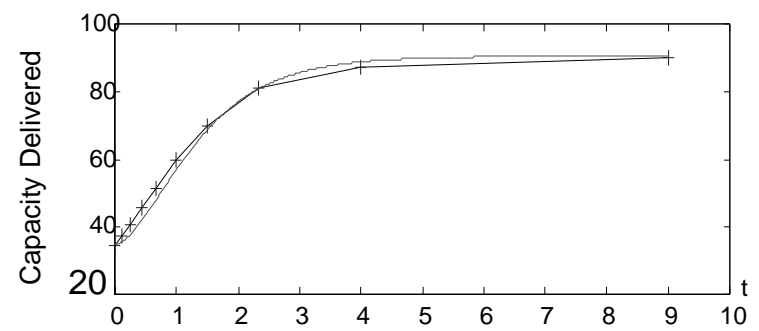

Figure 5. Relationship between the capacity recovery effect and ratio of the rest time to the discharge time for a $\mathrm{Li}$-ion battery.

\subsubsection{Determining $\rho^{\prime}(s, a)$}

As stated previously, $\rho^{\prime}(s, a)$ represents the transition rate of the battery from state $b_{i}$ to $b_{i-1}, i=1, \cdots, N$, when the SP stays in state $S$ and action $a$ is chosen. It can be formulated as

$$
\rho^{\prime}(s, a)=\frac{p o w^{\prime}(s, a)}{(1-\beta(s, a)) \cdot C / N}
$$

where $C$ is the full energy capacity of the battery. Here $\beta(s, a)$ captures the rate capacity of the battery, $0<\beta(s, a)<1$. The SP state $s$ and the chosen action $a$ determine the current drawn from the battery, i.e. determine the value of $\beta(s, a)$. As shown in Figure 6 , under different discharge currents, the deliverable capacity of a secondary battery may be quite different (in Figure 6, this effect is more pronounced for battery $\mathrm{B} 1)$. So for different batteries, $\beta(s, a)$ may take different forms.

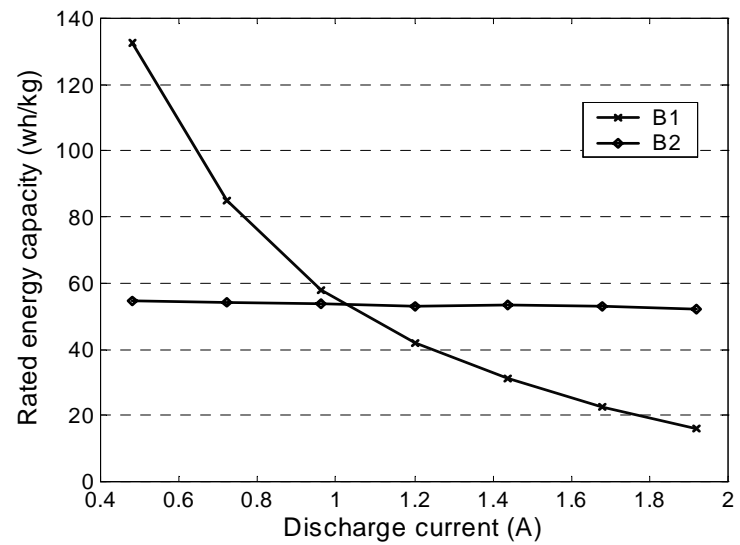

Figure 6. Current-capacity relations of two different batteries.

\subsubsection{Determining $\omega^{\prime}\left(b_{i}\right)$ and $v^{\prime}\left(b_{i}\right)$}

Transition rates $\omega^{\prime}\left(b_{i}\right)$ and $v^{\prime}\left(b_{i}\right)$ can be obtained from battery simulation results. Based on assumption (b), let $r_{i}(t), i=1, \ldots, N$, denote the expected recovered capacity during a time period $t$ (assuming that during this period, the recovery process is not interrupted by the discharge requests, i.e., the battery is not selected by the PS to power the SP), if at the beginning of this period the battery starts in state $b_{i}$. Define an $N \times 1$ vector $\mathbf{r}(t)=\left[\begin{array}{llll}r_{1}(t) & r_{2}(t) & \ldots & r_{N}(t)\end{array}\right]^{T}$, which satisfies the equation:

$$
\dot{\mathbf{r}}(t)=\mathbf{A r}(t)+\mathbf{b},
$$

where $\mathbf{A}$ is an $N \times N$ matrix: $\left(a_{i j}\right)_{N \times N}$, and

$$
a_{i j}=\left\{\begin{array}{lc}
-\left(\omega^{\prime}\left(b_{i}\right)+v^{\prime}\left(b_{i}\right)\right) & i=j \neq N, \\
\omega^{\prime}\left(b_{i}\right) & j=i+1, i \leq N-1, \\
0 & \text { otherwise. }
\end{array}\right.
$$

b is an $N \times 1$ vector: $\left(b_{i}\right)_{N \times 1}$, and

$$
b_{i}=\left\{\begin{array}{l}
\omega^{\prime}\left(b_{i}\right) \cdot C / N \quad i \leq N-1, \\
0 \quad i=N .
\end{array}\right.
$$

where $\mathrm{C}$ is the full energy capacity of the battery (defined in equation (2-2)).

The boundary condition is

$$
\mathbf{r}(0)=0
$$

The $\omega^{\prime}\left(b_{i}\right)$ and $v^{\prime}\left(b_{i}\right)$ can be determined as described below in a top-down manner:

1) Since $b_{N}$ represents a state of full capacity, $r_{N}(t) \equiv 0$.

2) Then $r_{N-1}(t)$ satisfies:

$$
\dot{r}_{N-1}(t)=-\left(\omega^{\prime}\left(b_{N-1}\right)+v^{\prime}\left(b_{N-1}\right)\right) r_{N-1}(t)+\frac{(N-1)}{N} \omega^{\prime}\left(b_{N-1}\right)
$$

using the boundary condition, we get

$$
r_{N-1}(t)=\left(1-\frac{1}{N}\right) \frac{\omega^{\prime}\left(b_{N-1}\right)}{\omega^{\prime}\left(b_{N-1}\right)+v^{\prime}\left(b_{N-1}\right)}\left(1-\exp \left(-\left(\omega^{\prime}\left(b_{N-1}\right)+v^{\prime}\left(b_{N-1}\right)\right) t\right)\right)
$$


Next, we do battery simulation: discharge the battery to $\frac{N-1}{N}$ of its original capacity, let it rest for a time period $t$, then fully discharge the battery. Next we change the value of $t$ and repeat the above steps. Proceeding in this way, we obtain a curve of the recovery capacity vs. rest time at the battery state $b_{N-1}$. Next, we choose a set of $\omega^{\prime}\left(b_{N-1}\right)$ and $v^{\prime}\left(b_{N-1}\right)$ that make the curve determined by equation (2-6) match the simulation curve best.

3) Since $r_{N-1}(t)$ is known, we can solve for $r_{N-2}(t)$ and determine $\omega^{\prime}\left(b_{N-2}\right)$ and $v^{\prime}\left(b_{N-2}\right)$ by repeating step 2). We repeat step 2) until $r_{1}(t)$ is obtained and $\omega^{\prime}\left(b_{1}\right)$ and $v^{\prime}\left(b_{1}\right)$ are thereby determined.

The steps can be simplified, if two conditions are satisfied: i) $\omega^{\prime}\left(b_{i}\right)$ is much less than $\rho^{\prime}(s, a)$, and ii) $v^{\prime}\left(b_{i}\right) \equiv v$, is independent of $b_{i}$. In this case, the expected recovered battery capacity related to the rest time may be approximated as follows,

$$
\begin{aligned}
& r_{i}(t)=\int_{0}^{t} \omega \tau \cdot v e^{-v \tau} d \tau \\
& =\frac{\omega}{v}\left(1-e^{-v t}-v t e^{-v t}\right)
\end{aligned}
$$

where $\omega=\frac{1}{k} \sum_{j=i}^{i+k-1} \omega^{\prime}\left(b_{j}\right)$, normally setting $k$ to 2 or 3 is adequate.

The solid curve shown in Figure 5 is the analytical results of the recovery effect given by this battery model when it is used to capture the real Li-ion battery of which the simulation result is also shown in Figure 5 as the curve marked with “+”). Indeed Figure 5 demonstrates that the analytical function describing the recovered capacity of the battery as a function of the rest time $t$ is very accurate (when compared with detailed battery simulation results).

A function ene $\left(b_{i}, b_{j}\right)$ is associated with each pair $\left(b_{i}, b_{j}\right)$. This function is defined as follows: ene $\left(b_{i}, b_{j}\right)= \pm C / N$, where $j=i \pm 1$. When $j=i-1$, ene $\left(b_{i}, b_{j}\right)>0$, represents the energy consumed when the battery transits from state $b_{i}$ to $b_{j}$. When $j=i+1$, ene $\left(b_{i}, b_{j}\right)<0$, represents energy recovered in the battery due to the battery relaxation process.

For a two-battery powered system, the generator matrix of the twobattery model is given as $G_{B}=G_{B 1} \otimes G_{B 2}$, where $\otimes$ is the tensor product of the two generator matrixes of battery B1 and B2 [15].

\subsection{Model of the Battery-Powered System}

We use five components: SR, SQ, SP, PS and BAT models, to construct the model of a power-managed, portable, battery-powered system. The state set is given by: $X=R \times Q \times S \times W \times B-\{$ invalid states $\}$. The invalid states include the states where SP is busy and SQ is empty. Thus the SYS state can be represented as a quintuple $x=(r, q, s, w, b)$, where $b=\left\{b^{(1)}, b^{(2)}\right\} \in B, b^{(1)} \in B_{1}, b^{(2)} \in B_{2}$.

The system action set $A_{s y s}$ is the union of the action set $A_{s}$ for the SP and the action set $A_{s w}$ for the PS. We use $G_{S Y S}(a)$ to represent the generator matrix of the system, where $a \in A_{s y s}$. Since the service requester is assumed to be independent of the other components, the generator matrix $G_{S Y S}(a)$ can be calculated as

$$
\begin{gathered}
G_{S Y S}(a)=G_{S R}(a) \oplus G_{S Q-S P-P S-B A T}(a), \\
G_{S P-P S}(a)=G_{S P}(a) \oplus G_{P S}(a),
\end{gathered}
$$

where the SQ-SP-PS-BAT denotes the joint CTMDP model of the SQ, SP, PS and BAT, and the SP-PS denotes the joint CTMDP model of the SP and PS.

Unfortunately, the Markovian processes of the SQ and the SP-PS, and the Markovian processes of the BAT and the SP-PS are both correlated. The SP-PS and the Battery are correlated in the sense that when the state of the SP-PS changes, the discharge rate of the Battery also changes. Based on our assumptions for the battery model, we can calculate each entry of the $G_{S Q-S P-P S-B A T}(a)$ as described below in detail.

Let $\sigma_{x, x^{\prime}}$ denote the transition rate of the system for going from state $x=(q, s, w, b)$ to state $x^{\prime}=\left(q^{\prime}, s^{\prime}, w^{\prime}, b^{\prime}\right)$.

1) If $b^{\prime}=b, \sigma_{x, x^{\prime}}$ is equal to $\sigma_{(s, w, q),\left(s^{\prime}, w^{\prime}, q^{\prime}\right)}$, which is the joint state transition rate of the SQ-SP-SW.

2) If $s^{\prime}=s$ and $q^{\prime}=q$, and if $w=w_{1}$ and $b^{(1)}=b_{i}$ and $b^{(1)}=b_{i-1}$ and $b^{(2)}=b^{(2)}$, then $\sigma_{x, x^{\prime}}$ is equal to $\rho_{B_{1}}(s, a)$, which is the discharge transition rate of battery $B_{1}$ from state $b_{i}$ to state $b_{i-1}$.

3) If $s^{\prime}=s$ and $q^{\prime}=q$, and if $w=w_{2}$ and $b^{(2)}=b_{i}$ and $b^{(2)}=b_{i-1}$ and $b^{(1)}=b^{(1)}$, then $\sigma_{x, x^{\prime}}$ is equal to $\rho_{B_{2}}(s, a)$, which is the discharge transition rate of battery $B_{2}$ from state $b_{i}$ to state $b_{i-1}$.

4) If $s^{\prime}=s$ and $q^{\prime}=q$, and if $w=w_{2}$ or the SP is in the sleep state, and if $b^{(1)}=b_{i}$ and $b^{(1)}=b_{i+1}$ and $b^{(2)}=b^{(2)}$, then $\sigma_{x, x^{\prime}}$ is equal to $\rho_{B_{1}}(s, a)$, which is the recovery transition rate of battery $B_{1}$ from state $b_{i}$ to state $b_{i+1}$.

5) If $s^{\prime}=s$ and $q^{\prime}=q$, and if $w=w_{1}$ or the SP is in the sleep state, and if $b^{(2)}=b_{i}$ and $b^{(2)}=b_{i+1}$ and $b^{(1)}=b^{(1)}$, then $\sigma_{x, x^{\prime}}$ is equal to $\rho_{B_{2}}(s, a)$, which is the recovery transition rate of battery $B_{2}$ from state $b_{i}$ to state $b_{i+1}$.

6) If $s^{\prime}=s$ and $q^{\prime}=q$, and if $w=w_{2}$ or the SP is in the sleep state, and if $b^{(1)}=b_{i}$ and $b^{(1)}=r s_{i}$ and $b^{(2)}=b^{(2)}$, then $\sigma_{x, x^{\prime}}$ is equal to $v_{B_{1}}(s, w)$, which is the transition rate of battery $B_{1}$ from state $b_{i}$ to state $r s_{i}$.

7) If $s^{\prime}=s$ and $q^{\prime}=q$, and if $w=w_{1}$ or the SP is in the sleep state, and if $b^{(2)}=b_{i}$ and $b^{(2)}=r s_{i}$ and $b^{(1)}=b^{(1)}$, then $\sigma_{x, x^{\prime}}$ is equal to $v_{B_{2}}(s, w)$, which is the transition rate of battery $B_{2}$ from state $b_{i}$ to state $r s_{i}$.

8) For any other transition, $\sigma_{x, x^{\prime}}$ is equal to 0. 


\section{SOLUTION METHOD}

\subsection{Cost Function}

The expected cost $\gamma_{x}^{a_{x}}$, which represents the expected energy delivered from the battery when the system is in state $x$ and action $a_{x}$ is chosen, is calculated as:

$$
\gamma_{x}^{a_{x}}=\sum_{x^{\prime}} p_{x, x^{\prime}}^{a_{x}} \cdot \operatorname{ene}\left(b, b^{\prime}\right)
$$

Let $f_{x}^{a_{x}}$ denote the frequency that the system will be in state $x$ and action $a_{x}$ is chosen. Let $\tau_{x}^{a_{x}}$ denote the expected time that the system will stay in state $x$ when action $a_{x}$ is chosen. Let $l q_{x}^{a_{x}}$ denote the waiting cost in the queue, $l q_{x}^{a_{x}}$ can be calculated as $l q_{x}^{a_{x}}=q_{x} \cdot \tau_{x}^{a_{x}}$.

\subsection{Objective function}

Our goal is to find an optimal policy to minimize the energy delivered from the batteries under the constraints on the average number of waiting requests in the queue and the request loss rate. Notice that a request issued by the SR is lost in (dropped by) the SQ if the queue is full when the request comes in. We formulate this problem as a linear program as follows:

$$
\text { Minimize }_{\left\{x^{\left.a_{x}\right\}}\right.}\left(\sum_{x} \sum_{a_{x}} f_{x}^{a_{x}} \gamma_{x}^{a_{x}}\right)
$$

subject to

$$
\begin{aligned}
& \sum_{a_{x}} f_{x}^{a_{x}}-\sum_{x^{\prime} \neq x} \sum_{a_{x^{\prime}}} f_{x^{\prime}}^{a_{x^{\prime}}} p_{x^{\prime}, x}^{a_{x^{\prime}}}=0, \forall x \in X \\
& \sum_{x} \sum_{a_{x}} f_{x}^{a_{x}} \tau_{x}^{a_{x}}=1 \\
& f_{x}^{a_{x}} \geq 0 \\
& \sum_{x} \sum_{a_{x}} f_{x}^{a_{x}} l q_{x}^{a_{x}}<D \\
& \sum_{x} \sum_{a_{x}} f_{x}^{a_{x}} \tau_{x}^{a_{x}} \delta\left(q_{x}^{a_{x}}, Q\right)<P_{\text {req_block }}
\end{aligned}
$$

where $\delta(x, y)=\left\{\begin{array}{ll}1, & \text { if } x=y ; \\ 0, & \text { otherwise. }\end{array}\right.$.

The last constraint ensures that the probability that the queue becomes full should be less than a preset threshold. It is our way of controlling request loss rate in the system.

\section{EXPERIMENTAL RESULTS}

It has been demonstrated that DPM techniques based on Markovian decision process outperform heuristic policies, when not considering the characteristics of the batteries [14]. To compare the effects of different power management policies on the battery service lifetime, in this experimental setup, we use the policy obtained in [4] to determine the behavior of the SP under a number of heuristic methods: M1-M4, see below. Notice that these heuristic methods do not intrinsically account for the battery effects as part of solving an integrated battery-aware power management problem, which is what we have proposed in this paper. We use the low-level simulator DUALFOIL [7] to simulate the batteries.
As shown in Figure 1, the experimental system contains a SR, a SP with its own SQ and two batteries. The details are as follows.

We use an input trace file to capture the statistical behavior of the SR. More precisely, the distribution of the input requests is a combination of the exponential and Pareto distribution as observed in [5].

The SP has six power states: \{busyl, idle1, busy2, idle2, waiting, sleeping $\}$. The busy1 and busy2 states are working states where the $\mathrm{SP}$ services the requests waiting in the queue. In the waiting or sleeping states, the SP does not service any requests. The only differences between the two states are as follows, 1) in the waiting state, the SP consumes power whereas in the sleeping state, it does not; 2) in the waiting state, the SP can return to a working state much faster than in the sleeping state. The idle states are in one-toone correspondence with the busy states. They are abstract states where new policy decisions are issued to the SP. Transition from busy to idle state is autonomous and instant. Since the DUALFOIL accepts current density as an input, in this experiment we express $\chi$, pow $(s)$, ene $\left(s_{i}, s_{j}\right)$ in terms of the current.

$$
\text { pow }=\left[\begin{array}{llllll}
0.9 & 1.6 & 0.9 & 1.6 & 0.3 & 0
\end{array}\right] \text { (unit: A), }
$$$$
\chi=\left[\begin{array}{cccccc}
\infty & 0 & 0.2 & 0 & 0 & 0 \\
0 & \infty & 0 & 0.33 & 0 & 9 \\
\infty & 0 & \infty & 1.68 & 1 & 0.5 \\
0 & \infty & 1.68 & \infty & 1 & 0.5 \\
0 & 0 & 0.454 & 0.454 & \infty & 1.5 \\
0 & 0 & 0.166 & 0.166 & 1.5 & \infty
\end{array}\right]
$$

$$
\text { ene } \left.=\left[\begin{array}{cccccc}
0 & \infty & 0 & \infty & \infty & \infty \\
\infty & 0 & \infty & 0 & \infty & \infty \\
0 & \infty & 0 & 0.017 & 0.056 & 0.11 \\
\infty & 0 & 0.017 & 0 & 0.056 & 0.11 \\
\infty & \infty & 0.25 & 0.25 & 0 & 5.3 \\
\infty & \infty & 1.69 & 1.69 & 0.51 & 0
\end{array}\right] \text { (unit }: A \cdot s\right) \text {. }
$$

The two batteries have different rate-capacity characteristics and recovery abilities. From Figure 6, we can see that in low current working state, busyl, battery B1 can deliver more energy than B2, while in high current working state, busy2, battery B2 can deliver more energy than B1. Figure 7 shows that battery B1 has a much stronger recovery ability than $\mathrm{B} 2$.

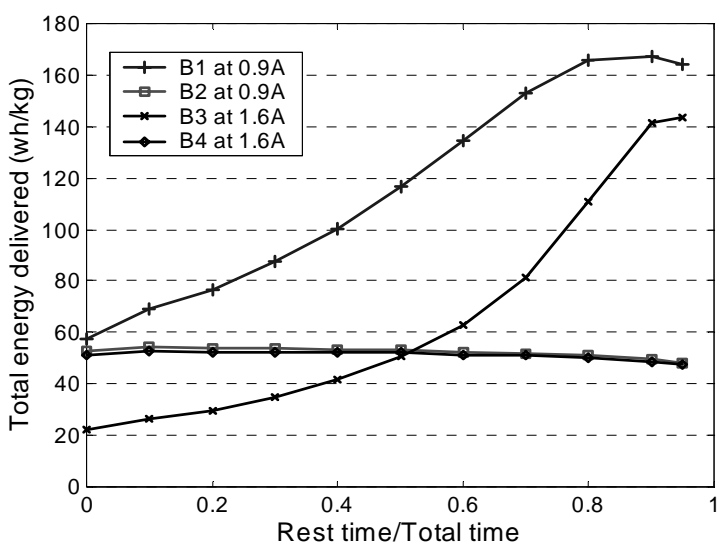

Figure 7. Recovery abilities of battery B1 and B2. 
We consider and compare four heuristic methods of battery management with our CTMDP-based policy:

M1: As in [13], we account for the rate-capacity characteristics of the battery, but do not consider the recovery effect and use a pre-assigned battery when the SP is in a particular state, e.g., we use battery B1 when the SP is running in the state busyl, while we use battery B2 when the SP is in state busy2.

M2: Similar to [16], we account for the recovery effect in battery but we do not consider the rate-capacity characteristics of batteries and switch between the two batteries (B1-B2) with a fixed frequency $(0.1 \mathrm{~Hz}$, as suggested in [16]).

M3: We use two batteries of type B1, switching between them (B1-B1) with a fixed frequency $(0.1 \mathrm{~Hz})$.

M4: We use two batteries of type B2, switching between them (B2-B2) with a fixed frequency $(0.1 \mathrm{~Hz})$.

Furthermore, we consider two battery replacement policies:

P1: As soon as a battery is completely consumed, it is immediately replaced with a new battery of the same type.

P2: The both batteries are replaced together and only after both have been completely used up. If only one battery is used up, the other battery will be used in all situations until it is also exhausted.

The experiment results are shown in Table 2. We can see that our method provides as much as $17 \%$ improvement over the heuristic methods.

Table 2. Experimental results for comparison

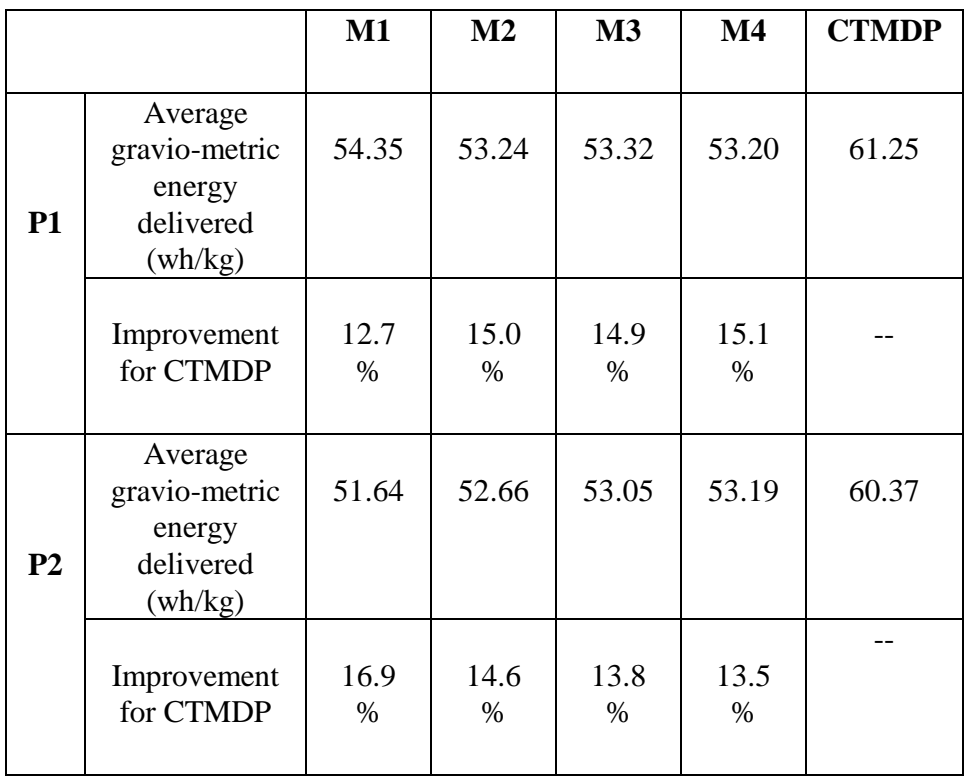

\section{CONCLUSION}

In this paper, a new stochastic model for the battery-powered portable electronic system is proposed based on continuous time Markovian decision processes. Two important battery characteristics: current-capacity and recovery effect are taken into account in this model. The battery-aware power management policy is solved as a Linear Programming problem. Experiment results demonstrate the effectiveness of this method.

\section{REFERENCES}

[1] M. Srivastava, A. Chandrakasan, and R. Brodersen, "Predictive system shutdown and other architectural techniques for energy efficient programmable computation," IEEE Trans. VLSI Syst., vol. 4, pp. 42-55, Mar. 1996.

[2] C-H. Hwang and A. Wu, "A predictive system shutdown method for energy saving of event-driven computation," Int. Conf. Computer-Aided Design, pp. 28-32, Nov. 1997.

[3] L. Benini, G. Paleologo, A. Bogliolo, and G. De Micheli, "Policy optimization for dynamic power management," IEEE Trans. Computer-AidedDesign, vol. 18, pp. 813-33, Jun. 1999.

[4] Qinru Qiu, Qing Wu and Massoud Pedram, "Stochastic modeling of a power-managed system-construction and optimization," Computer-Aided Design of Integrated Circuits and Systems, IEEE Transactions on, pp. 1200-1217, Oct. 2001.

[5] Simunic T, Benini L, Glynn P, De Micheli G. "Event-driven power management," Computer-Aided Design of Integrated Circuits and Systems, IEEE Transactions on, Vol. 20, pp. 840857, Jul. 2001.

[6] M. Pedram and Q. Wu, "Design Considerations for batterypower electronics," DAC 1999, pp. 861-866, 1999.

[7] M. Doyle, T.F. Fuller, and J. Newman, "Modeling of galvanostatic charge and discharge of the lithium/polymer/insertion cell", J. Electrochem Soc., Vol. 141, No. 1, pp. 1-9, Jan. 1994.

[8] Thomas F. Fuller, Marc Doyle and John Newman, "Relaxation phenomena in lithium-ion-insertion cells," J.Electrochem. Soc., Vol. 141, No. 4, Apr. 1994.

[9] Daler Rakhmatov and Sarma Vrudhula, "Time-to-failure estimation for batteries in portable electronic systems," ISLPED 2001, pp. 88-91, 2001.

[10] L. Benini, G. Castelli, A. Macii, E. Macii, M. Poncino, and R.Scarsi, "A discrete-time battery model for high-level power estimation," Proc. DATE, 2000.

[11] Carla-Fabiana Chiasserini, Ramesh R. Rao, "Energy Efficient Battery Management," IEEE journal of selected areas in communications, Vol. 19, No.7, Jul. 2001.

[12] L. Benini, G. Castelli, A. Macii, E. Macii, M. Poncino, R. Scarsi, "Extending lifetime of portable systems by battery scheduling," Proceedings of Design Automation and Test in Europe, pp. 197-201, 2001.

[13] Qing Wu, Qinru Qiu and Massoud Pedram, "An Interleaved Dual-battery Power Supply for Battery-Operated Electronics," DAC 2000, pp. 387-390, 2000.

[14] Qinru Qiu, Stochastic Modeling of a Power-Managed System: Construction and Optimization, Dissertation, Dec. 2000.

[15] U. N. Bhat, Elements of Applied Stochastic Processes. New York: Wiley, 1984.

[16] L. Benini, G. Castelli, A. Macii, R. Scarsi, "Batterydriven dynamic power management," IEEE Design \& Test of Computers, Vol. 18, pp. 53-60, 2001. 